Provided for non-commercial research and education use. Not for reproduction, distribution or commercial use.

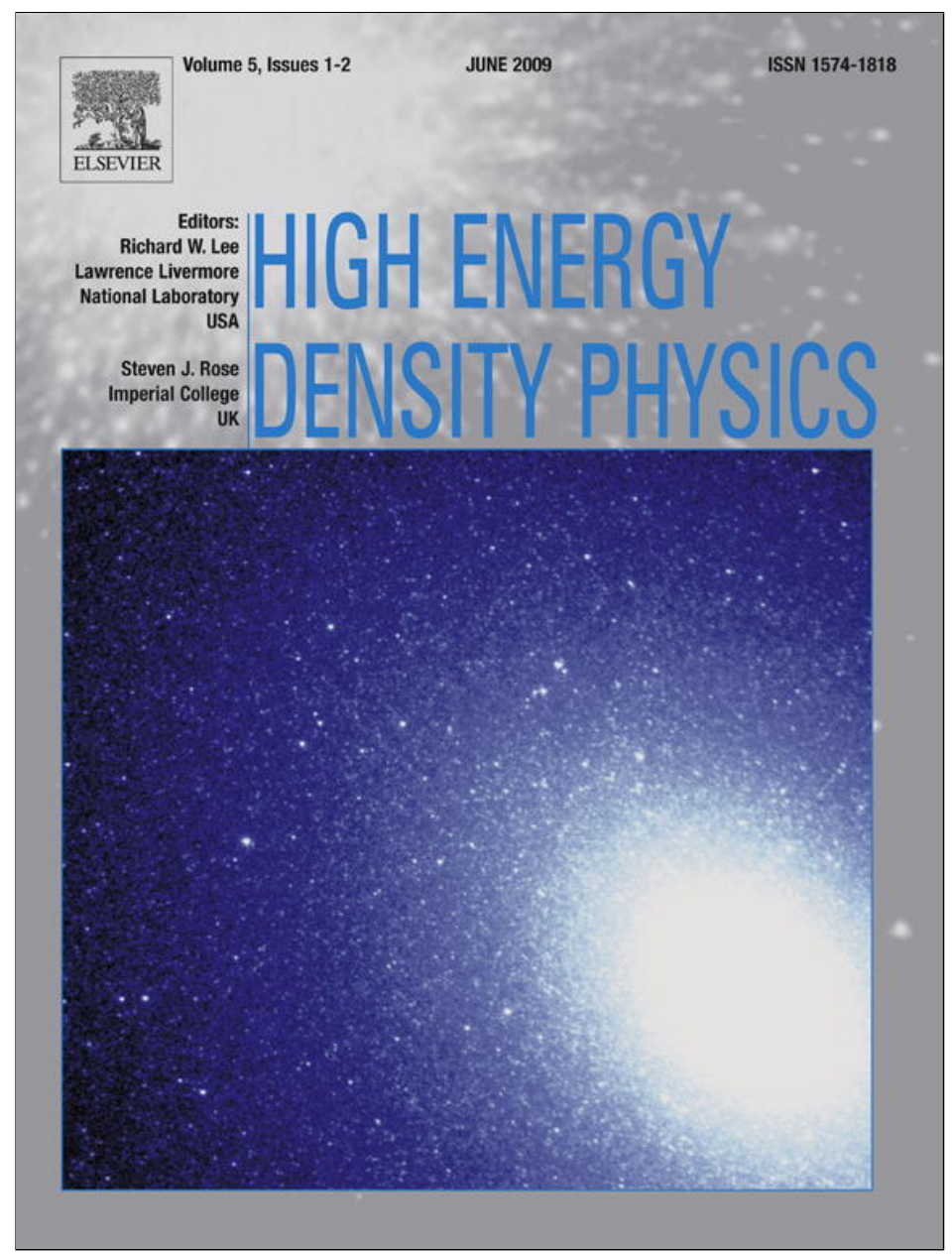

This article appeared in a journal published by Elsevier. The attached copy is furnished to the author for internal non-commercial research and education use, including for instruction at the authors institution and sharing with colleagues.

Other uses, including reproduction and distribution, or selling or licensing copies, or posting to personal, institutional or third party websites are prohibited.

In most cases authors are permitted to post their version of the article (e.g. in Word or Tex form) to their personal website or institutional repository. Authors requiring further information regarding Elsevier's archiving and manuscript policies are encouraged to visit:

http://www.elsevier.com/copyright 


\title{
Low-frequency conductivity in the average-atom approximation
}

\author{
W.R. Johnson* \\ Department of Physics, 225 Nieuwland Science Hall, University of Notre Dame, Notre Dame, IN 46556, United States
}

\section{A R T I C L E I N F O}

\section{Article history:}

Received 3 March 2009

Accepted 3 March 2009

Available online 21 March 2009

\section{PACS:}

51.70.+f

52.25.Mq

32.70.Cs

32.80.Fb

Keywords:

Plasma

Conductivity

Dielectric function

Index of refraction

Dispersion relations

$\mathrm{X}$-ray interferometry

\begin{abstract}
A B S T R A C T
The quantum-mechanical average-atom model is reviewed and applied to determine scattering phase shifts, mean-free paths, and relaxation times in warm-dense plasmas. Static conductivities $\sigma$ are based on an average-atom version of the Ziman formula. Applying linear response to the average-atom model leads to an average-atom version of the Kubo-Greenwood formula for the frequency-dependent conductivity $\sigma(\omega)$. The free-free contribution to $\sigma(\omega)$ is found to diverge as $1 / \omega^{2}$ at low frequencies; however, considering effects of multiple scattering leads to a modified version of $\sigma(\omega)$ that is finite and reduces to the Ziman formula at $\omega=0$. The resulting average-atom version of the Kubo-Greenwood formula satisfies the conductivity sum rule. The dielectric function $\epsilon(\omega)$ and the complex index of refraction $n(\omega)+i \kappa(\omega)$ are inferred from $\sigma(\omega)$ using dispersion relations. Applications to anomalous dispersion in laser-produced plasmas are discussed.
\end{abstract}

() 2009 Elsevier B.V. All rights reserved.

\section{Average-atom and static conductivity}

Let us briefly reprise the average-atom model, which is a quantum-mechanical version of the temperature-dependent Thomas-Fermi theory of a plasma introduced sixty years ago by Feynman etal. [1]. In the average-atom model, the plasma is divided into neutral spherical cells, each containing a single nucleus (charge $Z$ ) and $Z$ electrons. The radius of each cell is the Wigner-Seitz (WS) radius, determined from the material density $\rho_{\mathrm{m}}(\mathrm{gm} / \mathrm{cc})$, the atomic weight $A(\mathrm{gm} / \mathrm{mol})$, and Avogadro's number $\mathcal{A}=6.023 \times 10^{23}$, by $R_{\mathrm{WS}}=(3 \Omega / 4 \pi)^{1 / 3}$, where $\Omega=A / \mathcal{A} \rho_{\mathrm{m}}$ is the cell volume. Individual electrons (bound and continuum) inside a neutral cell are assumed to move in a self-consistent potential. Outside the cell boundaries the potential vanishes. The continuum electrons penetrate the cell boundary and move into the region between atoms where the electron density approaches a constant value $\rho_{0}$ determined by the temperature $T$ and chemical potential $\mu$. In the quantum-mechanical version of the average-atom model, the density oscillates about $\rho_{0}$ outside the cell with a small and ever decreasing amplitude [2]. To insure electrical neutrality, it is

\footnotetext{
* Tel.: +1 5746316651; fax: +1 5746315952 .

E-mail address: johnson@nd.edu

URL: http://www.nd.edu/ johnson
}

necessary to assume a uniform positive background charge $\rho_{+}$that precisely cancels $\rho_{0}$. The average atom floats in this positive "jellium" sea. The average ionic charge of sea is $Z^{*}=\rho_{+} \Omega$. The picture that evolves is an average atom of nuclear charge $Z$ with $Z$ bound and continuum electrons moving self-consistently inside a sphere of radius $R_{\mathrm{WS}}$; outside is a neutral plasma consisting of electrons (density $\rho_{0}$ ) balanced by positive sea of ions (charge $Z^{*}$ ). The electron density is determined using the quantum-mechanical self-consistent field method. The average-atom model introduced here is a nonrelativistic version of Liberman's Inferno model [3] and is very similar to the model described previously by Blenski and Ishikawa [4].

In the quantum-mechanical model, each electron is assumed to satisfy the central-field Schrödinger equation

$\left[\frac{p^{2}}{2 m}-\frac{Z}{r}+V(r)\right] u_{a}(\boldsymbol{r})=\epsilon u_{a}(\boldsymbol{r})$

where $a=(n, l)$ for bound stares or $(\epsilon, l)$ for continuum states. The wave function $u_{a}(\boldsymbol{r})$ is decomposed in a spherical basis as

$u_{a}(\boldsymbol{r})=\frac{1}{r} P_{a}(r) Y_{l_{a} m_{a}}(\widehat{r}) \chi_{\sigma_{a}}$,

where the bound $P_{n l}(r)$ and continuum $P_{\epsilon l}(r)$ radial functions are normalized as 
$\int_{0}^{\infty} \mathrm{d} r P_{n l}(r) P_{n^{\prime} l}(r)=\delta_{n n^{\prime}}, \quad \int_{0}^{\infty} \mathrm{d} r P_{\epsilon l}(r) P_{\epsilon^{\prime} l}(r)=\delta\left(\epsilon-\epsilon^{\prime}\right)$.

The self-consistent potential consists of two parts: $V(r)=V_{\mathrm{dir}}+V_{\mathrm{xc}}$. The direct part of the potential is obtained from

$\nabla^{2} V_{\text {dir }}=-4 \pi \rho$

where the electron density $\rho=\rho_{\mathrm{b}}+\rho_{\mathrm{c}}$ has contributions from both bound and continuum electrons. In the present work, the exchange-correlation potential is taken to be the Kohn-Sham (exchange-only) potential

$V_{\mathrm{xc}}=-\left(\frac{3}{\pi} \rho(r)\right)^{1 / 3}$.

The bound-state contribution to the density is

$4 \pi r^{2} \rho_{\mathrm{b}}(r)=\sum_{n l} \frac{2(2 l+1)}{1+\exp \left[\left(\epsilon_{n l}-\mu\right) / k T\right]} P_{n l}(r)^{2}$

where $(n, l)$ ranges over all subshells. Contributions to the density from continuum electrons are given by

$4 \pi r^{2} \rho_{\mathrm{c}}(r)=\sum_{l} \int \mathrm{d} \epsilon \frac{2(2 l+1)}{1+\exp [(\epsilon-\mu) / k T]} P_{\epsilon l}(r)^{2}$.

Finally, the chemical potential $\mu$ is chosen to insure electric neutrality:

$Z=\int_{r<R} \rho(r) \mathrm{d}^{3} r \equiv \int_{0}^{R} 4 \pi r^{2} \rho(r) \mathrm{d} r$.

Eqs. (1)-(8) stated above are solved self-consistently to give the chemical potential $\mu$, the self-consistent potential $V(r)$ and the electron density $\rho(r)$.

As an illustration of the present average-atom code, consider aluminum at density $\rho_{\mathrm{m}}=0.27 \mathrm{gm} / \mathrm{cc}$ and temperature $T=5 \mathrm{eV}$. The WS radius is $R_{\mathrm{WS}}=6.44$ a.u. and the chemical potential is $\mu=-0.382$ a.u. In Fig. 1 , we show bound-state and continuum contributions to the radial density along with the effective charge

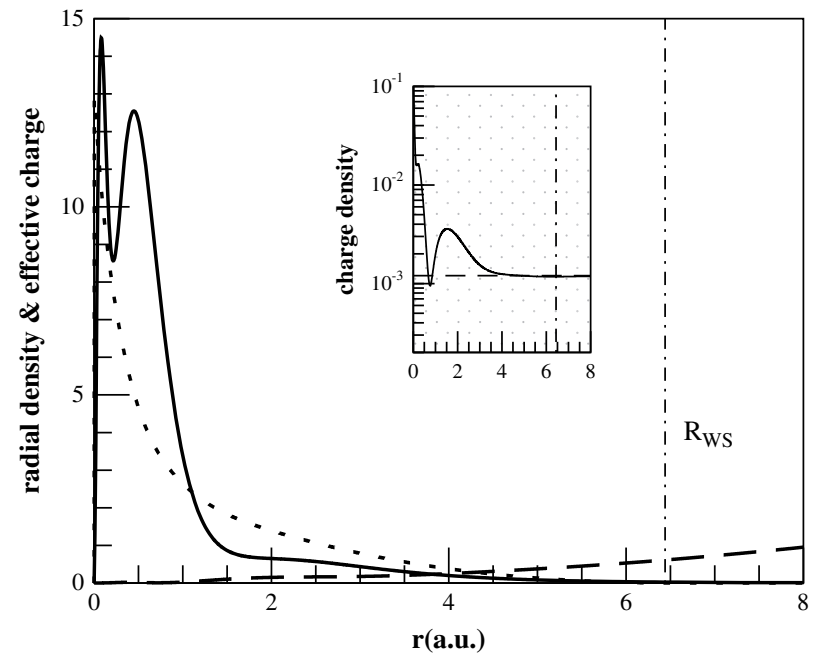

Fig. 1. Contributions to the radial electron density in an aluminum plasma, density $=0.27 \mathrm{gm} / \mathrm{cc}, T=5 \mathrm{eV}$. Bound-electron radial density $4 \pi \mathrm{r}^{2} \rho_{\mathrm{b}}(r)$ (solid), continuum radial density $4 \pi r^{2} \rho_{\mathrm{c}}(r)$ (dashed), and effective charge $Z_{\mathrm{eff}}(r)$ (dotted). Inset: continuum density $\rho_{\mathrm{c}}(r)$ (solid), background density $\rho_{0}$ (dashed).
$Z_{\mathrm{eff}}(r)$, related to the self-consistent potential by $V(r)=-Z_{\mathrm{eff}}(r) / r$. In the inset to the figure, the continuum contribution to the electron density $\rho_{\mathrm{c}}(r)$ is compared with the free-electron background density $\rho_{0}$ calculated using the same chemical potential. The density merges smoothly into the background outside the WS sphere.

In Fig. 2, we present phase shifts for partial waves with $l=0-6$ for energies $E \leq 30 \mathrm{eV}$, calculated in the average-atom potential illustrated in Fig. 1. From these phase shifts, we can calculate the scattering amplitude

$f(\theta)=\frac{1}{2 i p} \sum_{l}\left(\mathrm{e}^{2 i \delta_{l}}-1\right) P_{l}(\cos \theta)$,

describing elastic scattering of the continuum electrons on the average atom, considered as an impurity in the jellium sea. The corresponding elastic scattering cross-section is given by $\sigma_{\mathrm{el}}(\theta)=|f(\theta)|^{2}$. The transport cross-section $\sigma_{\mathrm{tr}}(p)$, which is important in determining the electric conductivity is given by

$\sigma_{\mathrm{tr}}(p)=\int(1-\cos \theta) \sigma_{\mathrm{el}}(\theta) \mathrm{d} \Omega=\frac{4 \pi}{p^{2}} \sum_{l=0}^{\infty}(l+1) \sin ^{2}\left(\delta_{l+1}-\delta_{l}\right)$.

Classically, the conductivity of a plasma is given by the Drude formula

$\sigma=\frac{\rho e^{2}}{m} \tau \quad$ (Drude Formula)

where $e$ and $m$ are the electron's charge and mass. Here, $\rho$ is the electron density and $\tau$ is the relaxation time (mean time between collisions). The relaxation time $\tau_{p}$, which depends on the electron's momentum $p$, is evaluated at the Fermi momentum and can be determined from the mean-free-path $\Lambda_{p}$ by $\tau_{p}=\Lambda_{p} / v$, where $v$ is the electron velocity. The mean-free-path is, in turn, related to the transport cross-section $\sigma_{\mathrm{tr}}(p)$ by $\Lambda_{p}=\Omega / \sigma_{\mathrm{tr}}(p)$, where $\Omega$ is the volume per scattering center. The Ziman formula [5],

$\sigma=\frac{2 e^{2}}{3} \int \frac{\mathrm{d}^{3} p}{(2 \pi)^{3}}\left(-\frac{\partial f}{\partial E}\right) v^{2} \tau_{p} \quad$ (Ziman formula)

provides a generalization of the Drude formula based on arguments from kinetic theory. Inasmuch as the relaxation time $\tau_{p}$ for an

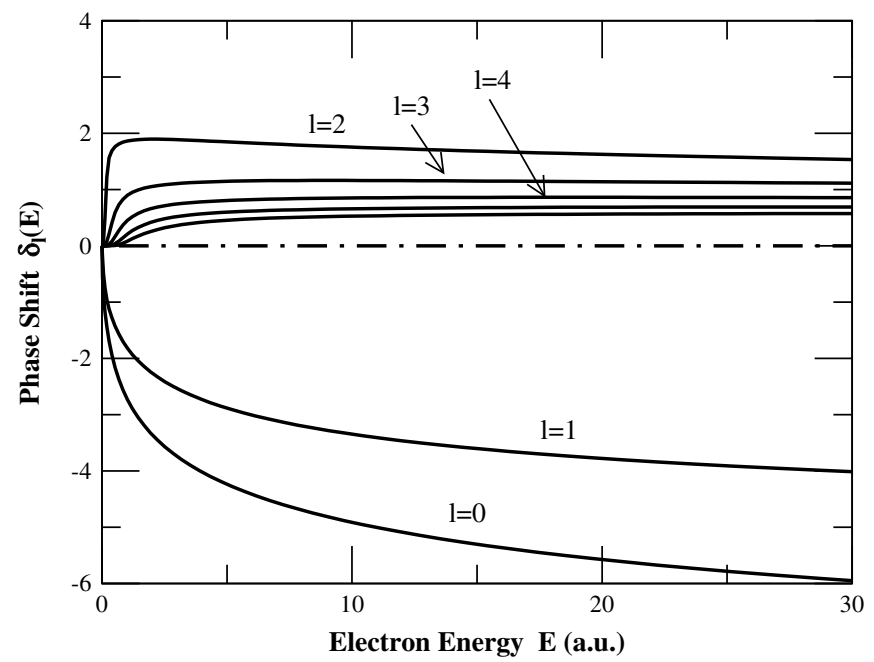

Fig. 2. Phase shifts for partial waves with $l \leq 6$ in an aluminum plasma with density $0.27 \mathrm{gm} / \mathrm{cc}$, and $T=5 \mathrm{eV}$. 
average atom can be determined directly in terms of average-atom phase shifts, the Ziman formula gives the static conductivity of a plasma within the average-atom framework. In Fig. 3, we compare average-atom calculations of the conductivity of an aluminum plasma at $T=20,000 \mathrm{~K}$ and densities ranging from 0.01 to $3 \mathrm{gm} / \mathrm{cc}$ with experimental measurements from Refs. [6-8]. The theoretical conductivity is in qualitative agreement with experiment but differs by as much as an order of magnitude near the center of the plot.

\section{Kubo-Greenwood formula (infrared catastrophe)}

The linear response of an average atom to an applied timedependent electric field $\boldsymbol{E}(t)=F \widehat{\boldsymbol{z}} \sin \omega t$ leads to an average-atom version of the Kubo-Greenwood formula [9-11] for the frequencydependent conductivity $\sigma(\omega)$. We describe the field by the vector potential

$\boldsymbol{A}(t)=\frac{F}{\omega} \widehat{\boldsymbol{z}} \cos \omega t$,

leading to the following time-dependent Schrödinger equation for electron orbitals:

$\left[T_{0}+V(r)-\frac{e F}{\omega} v_{z} \cos \omega t\right] \psi_{i}(\boldsymbol{r}, t)=i \frac{\partial}{\partial t} \psi_{i}(\boldsymbol{r}, t)$.

We seek a solution to Eq. (13) in the form

$\psi_{i}(\boldsymbol{r}, t)=u_{i}(\boldsymbol{r}) \mathrm{e}^{-i \epsilon_{i} t}+w_{i}^{+}(\boldsymbol{r}) \mathrm{e}^{-i\left(\epsilon_{i}+\omega\right) t}+w_{i}^{-}(\boldsymbol{r}) \mathrm{e}^{-i\left(\epsilon_{i}-\omega\right) t}$,

where $u_{i}(\boldsymbol{r})$ is a solution to the time-independent average-atom Eq. (1) and where $w_{i}^{ \pm}(\boldsymbol{r})$ are small perturbations. We find

$\left[T_{0}+V(r)\right] u_{i}(\boldsymbol{r})=\epsilon_{i} u_{i}(\boldsymbol{r})$

$\left[T_{0}+V(r)-\left(\epsilon_{i} \pm \omega\right)\right] w_{i}^{ \pm}(\boldsymbol{r})=\frac{e F}{2 \omega} v_{z} u_{i}(\boldsymbol{r})$.

In writing these equations, we ignore the modification of the potential $V(r)$ induced by the field. Next, we expand the perturbed orbital in terms of unperturbed eigenfunctions as:

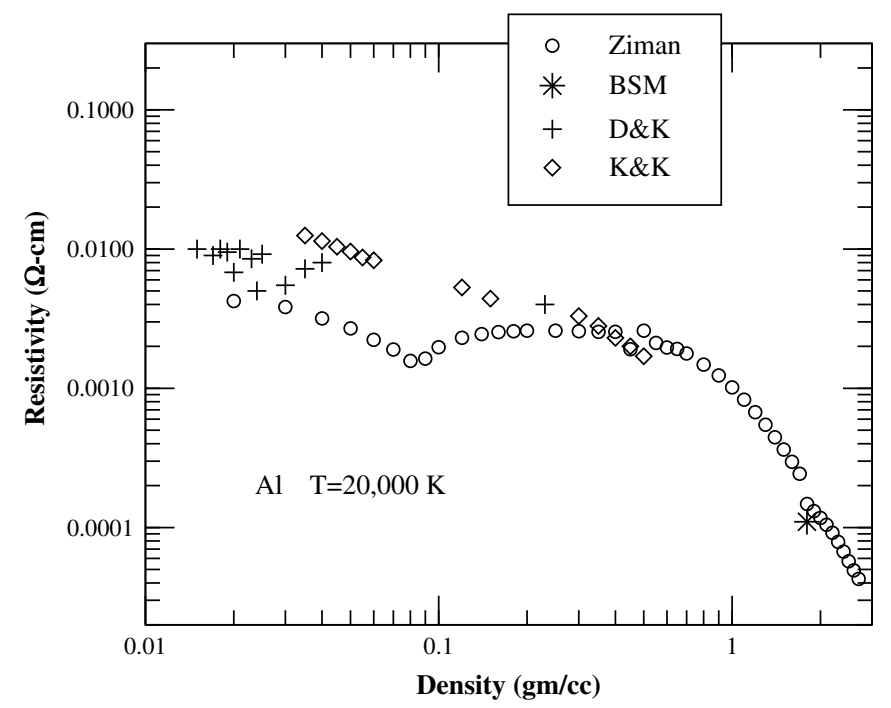

Fig. 3. Comparison of the static conductivity of an $\mathrm{Al}$ plasma at $T=20,000 \mathrm{~K}$ calculated using the average-atom Ziman formula with experimental results from Benage. Shanahan, and Murillo (BSM) [6], DeSilva and Katsouros (D \& K) [7], and Krisch and Kunze $(\mathrm{K} \& \mathrm{~K})[8]$. $w_{i}^{+}(\boldsymbol{r})=\sum_{j} X_{i}^{j} u_{j}(\boldsymbol{r}), \quad w_{i}^{-}(\boldsymbol{r})=\sum_{j} Y_{i}^{j} u_{j}(\boldsymbol{r})$.

From Eq. (15), we find

$X_{i}^{j}=\frac{e F}{2 \omega} \frac{\left\langle j\left|v_{z}\right| i\right\rangle}{\epsilon_{j}-i \eta-\epsilon_{i}-\omega}, \quad Y_{i}^{j}=\frac{e F}{2 \omega} \frac{\left\langle j\left|v_{z}\right| i\right\rangle}{\epsilon_{j}-i \eta-\epsilon_{i}+\omega}$.

The current associated with the perturbed atom is

$$
\begin{aligned}
J_{z}(t) & =\frac{2 e}{\Omega} \sum_{i} f_{i}\left\langle\psi_{i}(t)\left|v_{z}\right| \psi_{i}(t)\right\rangle \\
& =\frac{2 e}{\Omega} \sum_{i} f_{i}\left[\left(\left\langle u_{i}\left|v_{z}\right| w_{i}^{+}\right\rangle+\left\langle w_{i}^{-}\left|v_{z}\right| u_{i}\right\rangle\right) \mathrm{e}^{-i \omega t}+\text { c.c. }\right] .
\end{aligned}
$$

In the above equation $\Omega$ is the volume/atom. The function $f_{i}$ is the Fermi distribution function for state $i$ which accounts for the initial state occupation in the average atom. The response current may be rewritten in terms of the expansion coefficients as

$$
\begin{aligned}
J_{z}(t)= & \frac{4 e}{\Omega} \sum_{i j} f_{i}\left[\Re\left(\left\langle i\left|v_{z}\right| j\right\rangle X_{i}^{j}+\left\langle j\left|v_{z}\right| i\right\rangle Y_{i}^{j \star}\right) \cos \omega t\right. \\
& \left.+\Im\left(\left\langle i\left|v_{z}\right| j\right\rangle X_{i}^{j}+\left\langle j\left|v_{z}\right| i\right\rangle Y_{i}^{j \star}\right) \sin \omega t\right] .
\end{aligned}
$$

The conductivity is determined by that part of the current $J_{\text {in }}$ that is in phase with the driving field, which is given by

$$
J_{\text {in }}(t)=\frac{4 e}{\Omega} \sum_{i j} f_{i} \Im\left(\left\langle i\left|v_{z}\right| j\right\rangle X_{i}^{j}+\left\langle j\left|v_{z}\right| i\right\rangle Y_{i}^{j \star}\right) \sin \omega t,
$$

The out-of-phase part of the current corresponds to a "reactive" response that does not dissipate energy. One finds that

$$
\begin{aligned}
\Im\left(\left\langle i\left|v_{z}\right| j\right\rangle X_{i}^{j}+\left\langle j\left|v_{z}\right| i\right\rangle Y_{i}^{j \star}\right)= & \frac{e F}{2 \omega} \sum_{j}\left(\pi \delta\left(\epsilon_{j}-\epsilon_{i}-\omega\right) \mid\left\langle i\left|v_{z}\right| j\right\rangle \|^{2}\right. \\
& \left.-\pi \delta\left(\epsilon_{j}-\epsilon_{i}+\omega\right)\left|\left\langle j\left|v_{z}\right| i\right\rangle\right|^{2}\right) .
\end{aligned}
$$

Interchanging indices $i \leftrightarrow j$ in the second term lead to $J_{\text {in }}(t)=\sigma(\omega)$ $E_{z}(t)$ with

$\sigma(\omega)=\frac{2 \pi e^{2}}{\omega \Omega} \sum_{i j}\left(f_{i}-f_{j}\right)\left|\left\langle j\left|v_{z}\right| i\right\rangle\right|^{2} \delta\left(\epsilon_{j}-\epsilon_{i}-\omega\right)$.

Eq. (22) is an average-atom version of the Kubo-Greenwood (KG) formula. Contributions to the KG formula arise from bound-bound, bound-free, and free-free transitions from initial states $i$ to final states $j$. The bound-bound and bound-free transitions are regular for all values of $\omega$; however, the free-free contributions, which are dominant at low frequencies, diverge as $1 / \omega^{2}$, as will be shown in the following paragraph.

The matrix element appearing in Eq. (22) when taken between free-particle states vanishes unless the initial-state and final-state momenta are identical:

$\left\langle p_{2}\left|v_{z}\right| p_{1}\right\rangle=\delta\left(\boldsymbol{p}_{2}-\boldsymbol{p}_{1}\right) v_{z}$

By contrast, the matrix element of $v_{z}$ between continuum states in a potential $V(r)$ is inversely proportional to the energy difference $E_{2}-E_{1}=\omega$. Thus, considering $V(r)$ in lowest-order perturbation theory, one easily shows that

$\left\langle p_{2}\left|v_{z}\right| p_{1}\right\rangle \rightarrow-\frac{1}{m \omega} q_{z} V(q)$, 
where $\boldsymbol{q}=\boldsymbol{p}_{2}-\boldsymbol{p}_{1}$ is the momentum transfer between initial and final states. With the aid of the relation between scattering amplitude and potential in lowest-order perturbation theory (Born approximation),

$f(\theta)=-\frac{m}{2 \pi} V(q)$,

one can rewrite the dipole matrix element between continuum states in a potential in terms of the scattering amplitude as

$\left\langle p_{2}\left|v_{z}\right| p_{1}\right\rangle=\frac{2 \pi}{m^{2} \omega} q_{z} f(\theta)$.

Eq. (26) can be extended to all-orders in perturbation theory. This well-known relation is important for resolving the infrared problem in QED as discussed in Ref. [12]. The infrared divergence is illustrated in Fig. 4 where we plot dipole matrix elements for freefree transitions $l_{i}=2 \rightarrow l_{f}=1$ and $l_{i}=2 \rightarrow l_{f}=3$ as functions $\omega$.

\section{3. "Proper" static limit and conductivity sum rule}

Squaring the dipole matrix element in Eq. (26), and averaging over directions, we obtain

$\left\langle\left|\left\langle p_{2}\left|v_{z}\right| p_{1}\right\rangle\right|^{2}\right\rangle=\frac{2}{3} \frac{(2 \pi)^{2}}{m^{3} \omega^{2}} p^{2}(1-\cos \theta) \sigma_{\mathrm{el}}(\theta)$.

where

$f_{1}-f_{2} \approx-\omega \frac{\partial f}{\partial E}$

This leads to the following approximation for the free-free contribution to the conductivity

$$
\begin{aligned}
\sigma(\omega) & \approx \frac{2}{3} \frac{e^{2}}{\omega^{2} \Omega} \int \frac{\mathrm{d}^{3} p}{(2 \pi)^{3}}\left(-\frac{\partial f}{\partial E}\right) v^{3} \sigma_{\operatorname{tr}}(p) \\
& =\frac{2 e^{2}}{3} \int \frac{\mathrm{d}^{3} p}{(2 \pi)^{3}}\left(-\frac{\partial f}{\partial E}\right) v^{2} \frac{1}{\omega^{2} \tau_{p}}, \quad \text { (Low-Freq K-G formula) }
\end{aligned}
$$

which diverges as $1 / \omega^{2}$. In Fig. 5 , this low-frequency approximation (shown in the dashed line) is seen to be in excellent agreement with numerical calculations based on Eq. (22) (shown in the solid line) over the entire range of frequencies where either contributes.

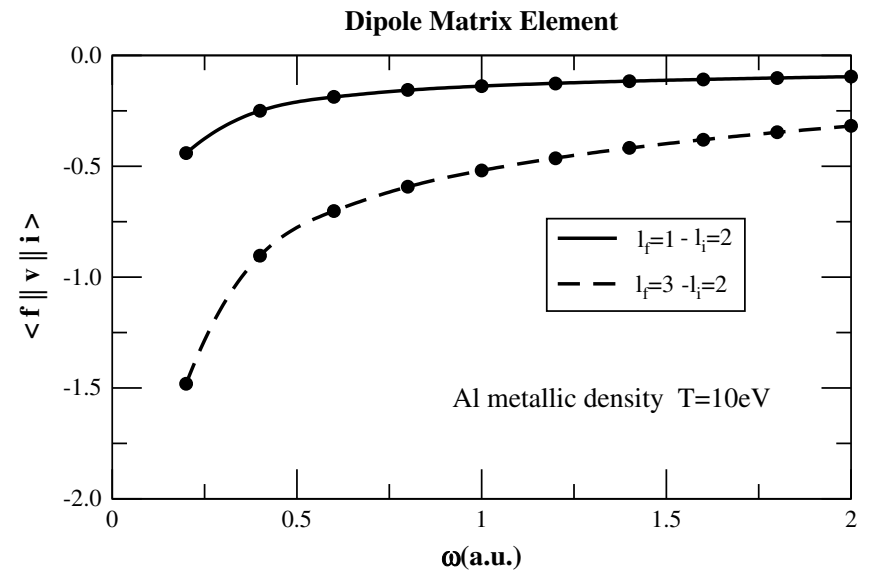

Fig. 4. Dipole matrix element between partial waves with $l_{i}=2$ and $l_{f}=1$ or 3 for Al at $T=10 \mathrm{eV}$ and metallic density.

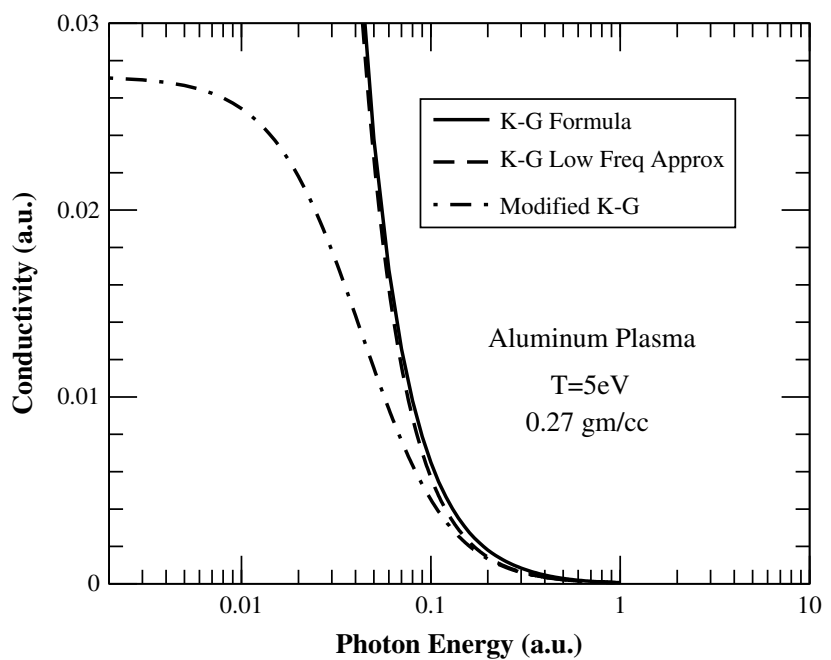

Fig. 5. The free-free contribution to the KG equation (solid line) is compared with its low-frequency approximation (dashed line). The modified expression for the free-free part of the KG equation is shown in the dot-dashed line.

It is interesting to note the similarities between the low-frequency approximation (29) and the Ziman formula (12): The factor $\tau_{p}$ in the Ziman formula is replaced by $1 /\left(\omega^{2} \tau_{p}\right)$ in the low-frequency KG formula.

The resolution of the differences between the low-frequency approximation to the KG formula and the Ziman formula was discussed in detail in Ref. [13] so an heuristic discussion should suffice here. First, we note that the asymptotic behavior of the averageatom scattering wave function is modified from a plane wave $\exp [i(\boldsymbol{p} \cdot \boldsymbol{r}-E t)]$ to a damped plane wave $\psi(\boldsymbol{p}, t) \rightarrow \exp [i(\boldsymbol{p} \cdot \boldsymbol{r}-E t)-$ $\left.t / \tau_{p}\right]$ when multiple scattering is considered. The effect of this modification is that the factor $1 / \omega$ in the free-free dipole matrix element Eq. (24) is replaced by

$\frac{1}{\omega} \rightarrow \frac{1}{\omega-i \tau_{p}}$.

With this in mind, the low-frequency KG formula becomes

$\sigma(\omega) \rightarrow \frac{2 e^{2}}{3} \int \frac{\mathrm{d}^{3} p}{(2 \pi)^{3}}\left(-\frac{\partial f}{\partial E}\right) v^{2} \frac{\tau_{p}}{\omega^{2} \tau_{p}^{2}+1}$.

(Modified KG Formula)

This modified KG formula reduces to the Ziman formula for $\omega \ll 1 / \tau_{p}$ and to the low-frequency KG formula when $\omega \gg 1 / \tau_{p}$. Thus, for "super-low" frequencies, those much smaller than the inverse relaxation time, the modified KG formula reduces to the expected Ziman limit. Fig. 5 compares the modified KG formula (dot-dashed line) with the KG formula (black line) and its lowfrequency limit (dashed line).

The modified KG formula for the free-free contribution to the conductivity satisfies the conductivity sum rule

$\int_{0}^{\infty} \sigma(\omega) \mathrm{d} \omega=\frac{\pi e^{2}}{3} \int \frac{\mathrm{d}^{3} p}{(2 \pi)^{3}} v^{2}\left(-\frac{\partial f}{\partial E}\right)=\frac{e^{2} \pi}{m} \int \frac{\mathrm{d}^{3} p}{(2 \pi)^{3}} f(E)=\frac{e^{2} \pi}{2 m} Z^{*}$,

where $Z^{*}$ is the background ionic charge. In Fig. 6, we show the three contributions to the frequency-dependent conductivity $\sigma(\omega)$, from free-free transitions, bound-bound transitions and bound-free transitions for aluminum at $T=5 \mathrm{eV}$, and density $0.27 \mathrm{gm} / \mathrm{cc}$. 

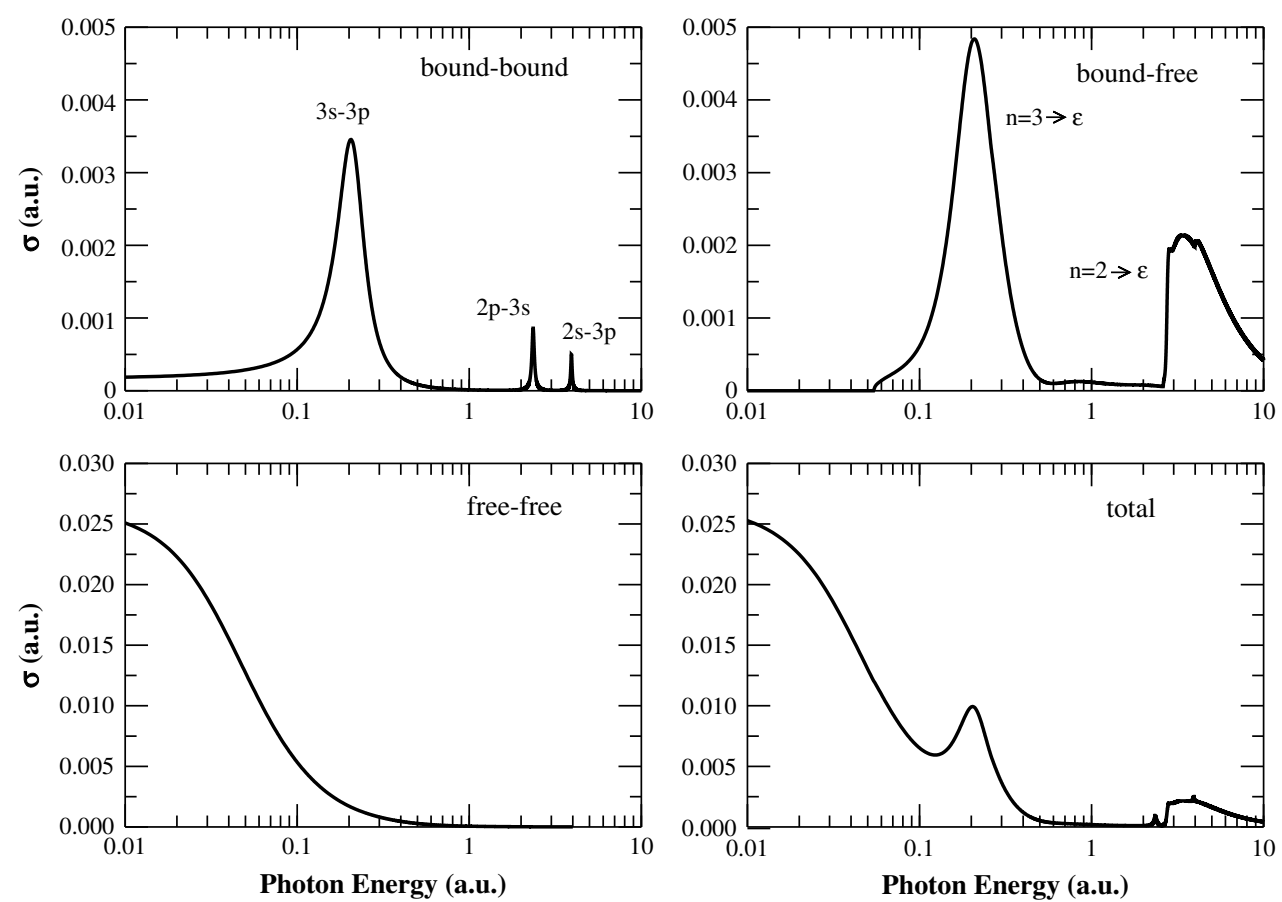

Fig. 6. Contributions to the frequency-dependent conductivity of an Al plasma ( $T=5 \mathrm{eV}$, density $0.27 \mathrm{gm} / \mathrm{cc}$ ). Lower left: free-free contribution; upper left: bound-bound contribution; upper right: bound-free contribution; lower right: resultant conductivity $\sigma(\omega)$.

\section{Application to plasma optics}

On general grounds of causality, one may infer the imaginary part of the dielectric function from its real part using a KramersKronig (KK) dispersion relation [14-17]. Owing to the close connection between the frequency-dependent conductivity and the dielectric function, the real and imaginary parts of the complex conductivity also satisfy a KK dispersion relation. This is particularly useful here since it permits us to obtain the imaginary part of the conductivity by a rather simple calculation. Moreover, because of the close connection between the conductivity and the dielectric function, we can obtain the latter directly. Once we have the dielectric function in hand, the optical properties of plasmas are completely characterized.

Given that $\sigma(\omega)$ is an even function of $\omega$ that is analytic in the upper half plane and falls faster than $1 / \omega$ for large $\omega$, we may write

$\Im \sigma\left(\omega_{0}\right)=-\frac{2 \omega_{0}}{\pi} f_{0}^{\infty} \frac{\Re \sigma(\omega)}{\omega^{2}-\omega_{0}^{2}} \mathrm{~d} \omega$.

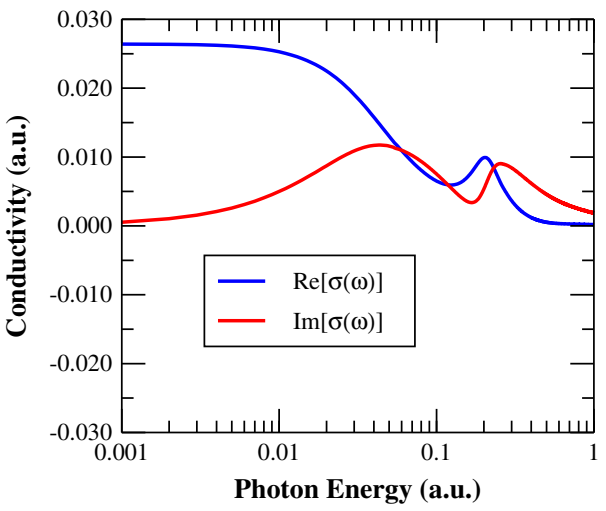

Applying this equation to the modified $\mathrm{K}-\mathrm{G}$ formula for $\operatorname{Re}[\sigma(\omega)]$, we find

$\Im \sigma(\omega)=\frac{2 e^{2}}{3} \int \frac{\mathrm{d}^{3} p}{(2 \pi)^{3}}\left(-\frac{\partial f}{\partial E}\right) v^{2} \frac{\omega \tau_{p}^{2}}{\omega^{2} \tau_{p}^{2}+1}$.

This leads to the following expression for free-free contribution to the complex conductivity $\sigma(\omega)$ as an analytic function of $\omega$,

$\sigma(\omega)=\frac{2 e^{2}}{3} \int \frac{\mathrm{d}^{3} p}{(2 \pi)^{3}}\left(-\frac{\partial f}{\partial E}\right) v^{2} \frac{\tau_{p}}{1-i \omega \tau_{p}}$.

Contributions to $\Im \sigma(\omega)$ from bound-bound and bound-free contributions are obtained from Eq. (33) by numerical integration. In Fig. 7, we plot real and imaginary parts of $\sigma(\omega)$ for an Al plasma $T=5 \mathrm{eV}$ and density $0.27 \mathrm{gm} / \mathrm{cc}$.

The (complex) plasma dielectric function is given in terms of the conductivity by the formula

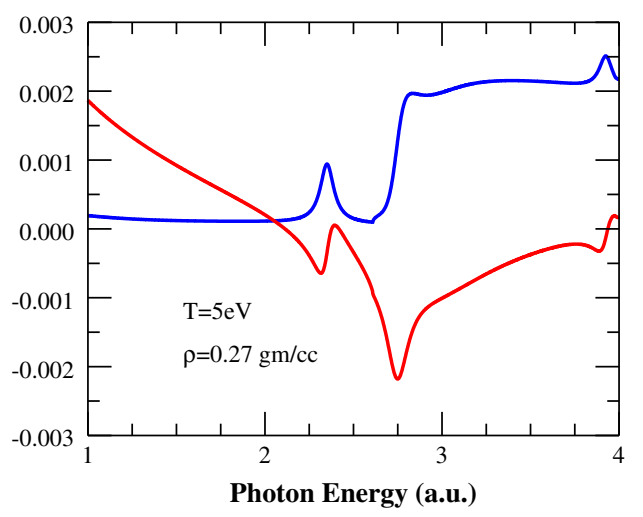

Fig. 7. The real and imaginary parts of the conductivity for an aluminum plasma, $T=5 \mathrm{eV}$ density $0.27 \mathrm{gm} / \mathrm{cc}$. The imaginary part is determined from the real part using Eq. (33). 


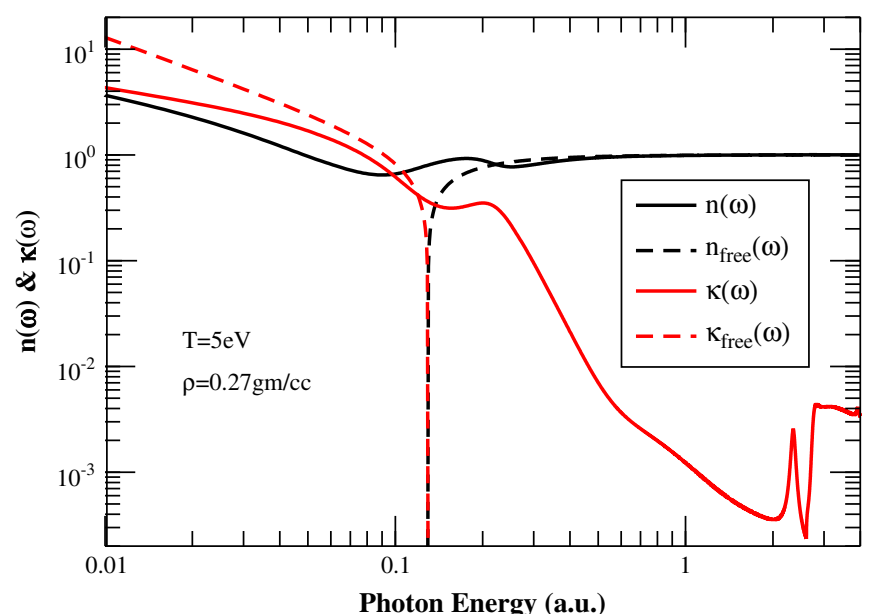

Fig. 8. Real and imaginary parts of the complex index of refraction $n(\omega)$ and $\kappa(\omega)$ for Al at $10 \mathrm{eV}$ and metallic density compared with their free-particle counterparts $n_{\text {free }}(\omega)$ and $\kappa_{\text {free }}(\omega)$.

$\epsilon_{r}(\omega)=1+4 \pi i \frac{\sigma(\omega)}{\omega}$.

We introduce the complex index of refraction $n+i \kappa$ through the equation

$n(\omega)+i \kappa(\omega)=\sqrt{\epsilon_{r}(\omega)}$.

It follows that

$n(\omega)=\sqrt{\frac{\sqrt{[\Re \epsilon(\omega)]^{2}+[\Im \epsilon(\omega)]^{2}}+\Re \epsilon(\omega)}{2}}$

$\kappa(\omega)=\sqrt{\frac{\sqrt{[\Re \epsilon(\omega)]^{2}+[\Im \epsilon(\omega)]^{2}}-\Re \epsilon(\omega)}{2}}$.

The two functions $n(\omega)$ and $k(\omega)$ are illustrated in Fig. 8, where they are plotted for the case of $\mathrm{Al}$ at $T=5 \mathrm{eV}$ and density $0.27 \mathrm{gm} / \mathrm{cc}$.

The classical free-electron expression for $\epsilon_{r}(\omega)$ is

$\epsilon_{r}(\omega)=1-\frac{\omega_{0}^{2}}{\omega^{2}}$

where $\omega_{0}$ is the plasma frequency $\omega_{0}^{2}=4 \pi e^{2} \rho_{0} / m$. It follows that at low frequencies $\omega<\omega_{0}, n_{\text {free }}=0$, while for $\omega>\omega_{0}, \kappa_{\text {free }}=0$. In Fig. 8 , we compare the average-atom results for $n(\omega)$ and $k(\omega)$ with the free-electron approximation for aluminum at $T=5 \mathrm{eV}$ and density $0.27 \mathrm{eV}$.

Instances where $n>1$, which are forbidden in the free-electron model, have been observed in interferograms of an $\mathrm{Al}$ plasma produced by a $14.7 \mathrm{~nm}$ Ni-like Pd soft X-ray laser at the Lawrence Livermore National Laboratory Comet laser facility [18]. Similar anomalies were observed in interference patterns of an Al plasma produced by a $13.9 \mathrm{~nm}$ Ni-like Ag laser at the Advanced Photon Research Center of the Japan Atomic Energy Research Institute [19]. The free-electron model leads to the conclusion that the square of the plasma frequency $\omega_{0}^{2}$ is negative! In Ref. [19] this behavior was correctly attributed to the $2 \mathrm{p}-3 \mathrm{~d}$ transition in $\mathrm{Al}^{+2}$. To examine such anomalous dispersion in terms of the average-atom model, we compare $n(\omega)-1$ predicted by the average-atom model with its free-electron counterpart $n_{\text {free }}-1$ in Fig. 9. The number of free electrons per ion from the average-atom model is $Z^{*}=1.38$ for the density and temperature illustrated. As can be seen in the figure,

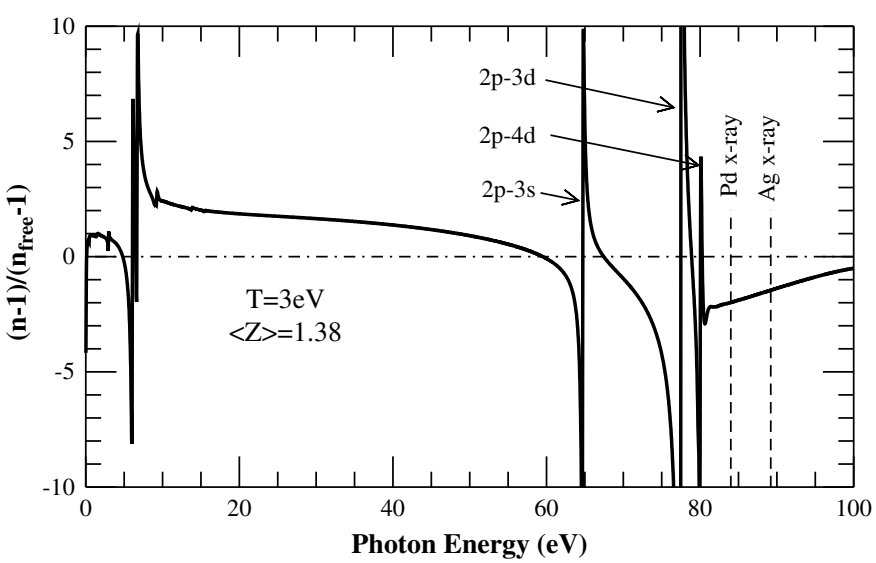

Fig. 9. The ratio of $n(\omega)-1$ to $n_{\text {free }}-1$ for $\mathrm{Al}$ at ion density $10^{20} / \mathrm{cc}$. The ratio is negative at the frequencies of $\mathrm{Ni}$ and $\mathrm{Ag}$ lasers explaining the effects of anomalous dispersion observed in experiments $[18,19]$.

the ratio is near -2 in a band of photon energies that includes those of the Pd and Ag lasers, which are indicated by the dashed lines at 84 and $89 \mathrm{eV}$. This explains the behavior seen experimentally. The primary effect on the dispersion integral in this region comes from $2 \mathrm{p}-2 \mathrm{~s}$ and $2 \mathrm{p}-3 \mathrm{~d}$ transitions and from photoionization of the $2 \mathrm{p}$ subshell (threshold at $80.2 \mathrm{eV}$ ).

\section{Conclusion}

In conclusion, the Kubo-Greenwood formula for $\sigma(\omega)$ applied to the average-atom model diverges as $1 / \omega^{2}$ at low frequencies. Including multiple scattering (finite relaxation time) leads to a modified version of the KG formula for the free-free contribution to $\sigma(\omega)$ that is finite and reduces to the static Ziman formula at $\omega=0$. The Kubo-Greenwood formula modified at low frequencies provides a useful approximation for studies of the optical properties of plasmas.

\section{Acknowledgements}

Work on the average-atom model and the average-atom version of the Ziman formula was initiated in collaboration with James Albritton. The average-atom version of the Kubo-Greenwood formula was developed in collaboration with Claude Guet and George Bertsch. The divergence-free model for the free-free contribution to the dynamic conductivity was developed in collaboration with Michael Kuchiev. Kwok-Tsang Cheng gave invaluable assistance in the development of reliable average-atom and conductivity codes. Finally, Joseph Nilsen is responsible for many recent applications of the Kubo-Greenwood codes to interpret space- and time-resolved interferograms in laser-produced plasmas and for comparing the average-atom model with alternative methods for studying plasma optics.

\section{References}

[1] R.P. Feynman, N. Metropolis, E. Teller, Equations of state of elements based on the generalized Fermi-Thomas theory, Phys. Rev. 75 (1949) 1561.

[2] J. Friedel, The distribution of electrons round impurities in monovalent metals, Philos. Mag. 43 (1952) 153.

[3] D.A. Liberman, Self-consistent field model for condensed matter, Phys. Rev. B 20 (1979) 4981.

[4] T. Blenski, K. Ishikawa, Pressure ionization in the spherical ion-cell model of dense-plasmas and a pressure formula in the relativistic Pauli approximation, Phys. Rev. E 51 (1995) 4869-4881.

[5] J.M. Ziman, A theory of the electrical properties of liquid metals: the monovalent metals, Philos. Mag. 6 (1961) 1013. 
[6] J.F. Benage, W.R. Shanahan, M.S. Murillo, Resistivity measurements of hot dense aluminum, Phys. Rev. Lett. 83 (1999) 2953-2956.

[7] A.W. DeSilva, J.D. Katsouros, Electrical conductivity of dense copper and aluminum plasmas, Phys. Rev. E 57 (1998) 5945-5951.

[8] I. Krisch, H.-K. Kunze, Measurements of electrical conductivity and the mean ionization state of nonideal aluminum plasmas, Phys. Rev. E 58 (1998) 6557-6564.

[9] R. Kubo, A general expression for the conductivity tensor, Can. J. Phys. 34 (1956) 1274.

[10] R. Kubo, Statistical-mechanical theory of irreversible processes. I. General theory and simple applications to magnetic and conduction problems, J. Phys. Soc. Jpn. 12 (1957) 570.

[11] D.A. Greenwood, The Boltzmann equation in the theory of electrical conduction in metals, Proc. Phys. Soc., London 715 (1958) 585.

[12] V.B. Berestetski, E.M. Lifshits, L. Pitayevski, Quantum Electrodynamics, Pergamon, Oxford, 1982.

[13] M.Y. Kuchiev, W.R. Johnson, Low-frequency plasma conductivity in the average-atom approximation, Phys. Rev. E 78 (2008) 026401
[14] R.de L. Kronig, On the theory of dispersion of X-rays, J. Opt. Soc. Am. 12 (1926) 547.

[15] H.A. Kramers, Some remarks of the theory of absorption and refraction of Xrays, Nature 117 (1926) 775.

[16] R.de L. Kronig, H.A. Kramers, La diffusion de la lumière par les atomes, Atti. Congr. Intern. Fisici. 2 (1927) 545.

[17] L.D. Landau, E.M. Lifshitz, Electrodynamics of Continuous Media, Pergamon, New York, 1984.

[18] J. Filevich, J.J. Rocca, M.C. Marconi, R.F. Smith, J. Dunn, R. Keenan, J.R. Hunter, S.J. Moon, J. Nilsen, J.H. Scofield, A. Ng, V.N. Shlyaptsev, Evidence of bound electron contributions to soft X-ray interferograms of dense plasmas, in: X-ray Lasers 2004, Proceedings of the 9th International Conference on X-ray Lasers, 2004.

[19] H. Tang, O. Guilbaud, G. Jamelot, D. Ros, A. Klisnik, D. Joyeux, D. Phalippou, M. Kado, M. Nishikino, M. Kishimoto, K. Sukegawa, M. Ishino, K. Nagashima, H. Daido, Diagnostics of laser-induced plasma with soft X-ray $(13.9 \mathrm{~nm})$ bimirror interference microscopy, Appl. Phys. B 78 (2004) 975-977. 